\title{
Size-dependent nonlinear elastic scaling of multiwalled carbon nanotubes
}

\author{
Irene Arias and Marino Arroyo* \\ LaCàN, Dept. Applied Mathematics 3, Universitat Politècnica de Catalunya, Barcelona 08034, Spain
}

(Dated: January 19, 2008)

\begin{abstract}
We characterize through large-scale simulations the nonlinear elastic response of multi-walled carbon nanotubes (MWNCNTs) in torsion and bending. We identify a unified law consisting of two distinct power-law regimes in the energy-deformation relation. This law encapsulates the complex mechanics of rippling and is described in terms of elastic constants, a critical length-scale and an anharmonic energy-deformation exponent. The mechanical response of MWCNTs is found to be strongly size-dependent, in that the critical strain beyond which they behave nonlinearly scales as the inverse of their diameter. These predictions are consistent with available experimental observations.
\end{abstract}

PACS numbers: $62.25 .+\mathrm{g}, 02.70 .-\mathrm{c}, 46.32 .+\mathrm{x}, 61.46 . \mathrm{Fg}$

Due to a unique combination of dimensions, geometry, mechanical, electronic and chemical properties, carbon nanotubes appear as an attractive component in nanoscale devices and nano-structured materials. Mechanically, the graphene wall of nanotubes is arguably the stiffest and strongest material in Nature. Under large tensile stress, CNTs fail irreversibly through plasticity or brittle fracture [1]. However, their geometric structure, the strength of carbon bonds, and a high degree of crystalline uniformity confers CNTs with a very rich nonlinearly elastic behavior over wide ranges of mechanical stress [2]. There is ample experimental evidence that CNTs can sustain dramatic geometric changes reversibly and cyclically [3]. The use of multiwalled carbon nanotubes (MWCNTs) as structural components in nano-devices has been demonstrated $[3,4]$. Recent CNTbased yarns and foam materials specifically exploit the resilience and ability to undergo extremely large deformations of MWCNTs [5, 6].

The experimentally observed deformation morphologies of CNTs have been interpreted in terms of the linearized theory of thin shell buckling [2], which studies the onset of bifurcations from homogeneous deformation states [7]. Beyond the bifurcation point, the postbuckling fully nonlinear regime governs the CNT mechanics. The rippling deformations (periodic wave-like deformation patterns) observed in bent nanotubes [3, 8], which have been shown to dramatically soften the effective response of thick MWNCTs [3], are a genuinely nonlinear phenomenon not explained by linearized buckling theory [9]. Theoretically, the understanding of the post-buckling behavior of thin elastic sheets is quite limited, and is the topic of current research $[10,11]$. The objective of the present study is to characterize through systematic largescale simulations the effective mechanical behavior of realistically large MWCNTs under torsion and bending, as probed in nano-devices and materials [3-6]. See [12] for an early related study resorting to a simplified model of MWNCTs

*Electronic address: marino.arroyo@upc.edu
We present coarse grained static atomistic simulations of MWCNTs. The bonded interactions are described through the Brenner potential [13], a standard for hydrocarbons known to underestimate the elastic moduli of graphene. We also use a modified version of this potential that maintains its functional form, the ground energy and the equilibrium bond length of graphene, and exhibits inplane and bending elastic moduli very close to $a b$ initio values [14]. The inter-wall van der Waals interactions are modelled with a Lennard-Jones graphitic potential [15] that produces smooth inter-wall tangential interactions, in agreement with experimental observations [16].

The coarse-grained computational method used here (see $[17,18]$ for the theory and implementation details) reduces the computational complexity of the atomistic models by two orders of magnitude without loss of accuracy [19], allowing us to perform systematically high fidelity simulations of realistically large MWCNTs as found in devices. The largest system in the present study contains about 31 million atoms, while state of the art molecular dynamics simulations of MWCNTs contain about 300,000 atoms [20]. We consider accurate boundary conditions for uniform bending, free of spurious boundary effects [21]. In torsion, the ends of all the walls are rotated relative to each other, modeling an effective load transfer between the walls at the ends suggested by experiments [4]. In all the simulations, we consider $(5,5),(10,10), \ldots,(5 n, 5 n)$ MWCNTs with $n=10$ to $n=40$ walls, i.e. thick tubes with minimal internal hollow space as often found in experiments.

To characterize the global mechanical response of MWCNTs seen as nano-beams, we study the strain energy vs deformation relation. As reported in [9], we observe for all the tested tubes and for wide ranges of deformation two distinct and robust regimes: an harmonic regime characterized by an energy-deformation power law with exponent 2, and an anharmonic (post-buckling) regime characterized by a different power-law with exponent $1<a<2$. These two regimes can be clearly observed in the log-log plots of Figs. 1 and 2. The latter behavior occurs as a consequence of a non-homogeneous deformation mode characterized by periodic ripples of the graphene walls $[3,8,9]$. In bending, there is experimen- 


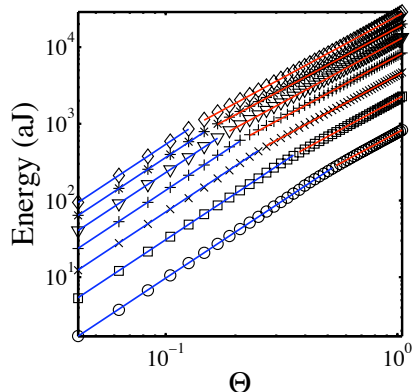

(a)

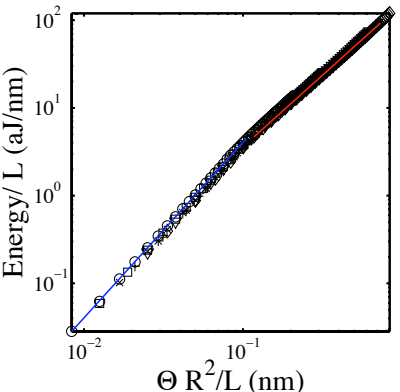

(b)

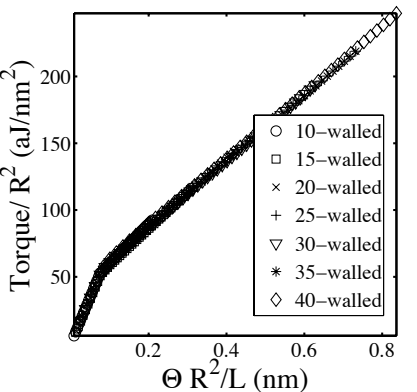

(c)

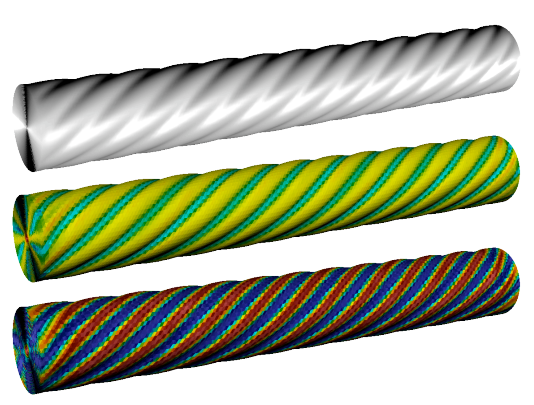

(d)

FIG. 1: Twisted MWCNTs. (a) Strain energy vs twisting angle log-log plots for various MWCNTs and (b) data collapse upon appropriate rescaling. The power-law fits with exponents 2 (blue) and 1.63 (red) are shown for illustration. (c) Rescaled torque vs twisting angle relation highlighting the unified law. (d) 35-walled CNT in torsion, deformed shape (top), Gaussian curvature map (middle, green is zero, red is positive, blue is negative), and energy density map (bottom, red is high, blue is low).

tal evidence of these two energy-deformation regimes [22]. The ripples in torsion are sequences of helicoidal ridges and furrows, while in bending the so-called Yoshimura or diamond pattern develops in the compressed part of the tubes. Rippling arises as an efficient mechanism to lower the elastic energy, and can be understood mechanically as distributed buckling. The van der Waals forces, irrelevant in the harmonic regime, are responsible for the collective buckling deformation of the walls in the postbuckling regime. In contrast with linear elasticity, the existence of two different power-laws breaks the scale invariance of the energy-deformation relationship. The cross-over buckling strain may be viewed as the critical point of a phase transition [21]. Despite the tubes are subject to severe deformations, the reported energydeformation behavior is reversible and free of noticeable hysteresis. The reversibility of the rippling deformations has been documented experimentally [3].

We study now the systematics of this behavior. It was hinted earlier that the anharmonic exponent showed a slight dependence on the number of walls [9]. The present study shows that this dependence is in fact an artifact of the boundary conditions, carefully avoided here [21]. As shown in Figs. 1 and 2, the anharmonic exponent is independent of the number of walls. The simulations show that it depends on the deformation mode and on the inter-atomic potential, as discussed later. The harmonic scaling can be easily explained in terms of the linear theory of elasticity. The stiffness of the graphene wall can be characterized in terms of two independent in-plane moduli (e.g. the Young's modulus $Y_{s}$ and the shear modulus $G_{s}$ ) and the bending modulus, denoted by $C_{b}$. We consider here surface moduli [14]. We consider MWCNTs of length $L$, with outer radius $R=15 n /(2 \pi) A_{0}$, where $A_{0}$ denotes the equilibrium bond length in graphene. In torsion, the graphene walls are subject to pure shear. The maximum shear occurs at the outer wall $\gamma=\Theta R / L$, where $\Theta$ denotes the applied rotation angle at the ends of the tube. By defining the torsional spring constant $K_{t}=\left[2 \pi G_{s} / R^{2}\right] \sum_{i=1}^{n} r_{i}^{3}$, where $r_{i}$ denote the radii of each wall, the blue fits in Fig. 1(a) follow from the formula for the strain energy $E=1 / 2 L K_{t} \gamma^{2}$. By approximating the sum in the formula for $K_{t}$ by an integral, and denoting the graphene wall spacing by $t$, we obtain $K_{t} \approx \pi G_{s} R^{2} /(2 t)$, hence

$$
\tilde{E}=E /\left(L R^{2}\right) \approx\left[\pi G_{s} /(4 t)\right] \gamma^{2} .
$$

This is the classical scaling of linear elastic torsion of beams, in which the deformation is characterized by a non-dimensional strain and the relation $\tilde{E}(\gamma)$ depends on the geometry of the cross section and the material properties, but does not depend on the size of the beam. However, since the energy-twisting angle relation for MWCNTs is not linearly elastic, the rescaling in Eq. (1) does not necessarily collapse the data for nanotubes of different sizes. Indeed, upon this rescaling, the curves for all MWCNTs collapse only in the harmonic regime, while the anharmonic branches do not follow a single law. In particular, we observe that the critical strain scales as $\gamma_{\mathrm{cr}} \propto R^{-1}$, which suggests using $\gamma R$ as the strain measure. The resulting rescaling for the energy-twisting angle relation in the harmonic regime follows from multiplying Eq. (1) by $R^{2}$

$$
\hat{E}=E / L \approx\left[\pi G_{s} /(4 t)\right](\gamma R)^{2} .
$$

Figure 1(b) shows the data collapse for all the tested nanotubes upon this rescaling, indicating that a unifying law is operative. This law is characterized by the elastic factor in Eq. (2), by the anharmonic exponent $a$, and by the transition deformation. The measure of deformation has dimensions of length, hence we denote the critical point by $\ell_{\mathrm{cr}}=\gamma_{\mathrm{cr}} R=\Theta_{\mathrm{cr}} R^{2} / L$. The unified law plotted in red and blue in Fig. 1(b) is

$$
E / L=\left[\pi G_{s} /(4 t)\right]\left\{\begin{array}{cll}
(\gamma R)^{2} & \text { for }|\gamma R| \leq \ell_{\mathrm{cr}} \\
\ell_{\mathrm{cr}}^{2-a}|\gamma R|^{a} & \text { for } & |\gamma R|>\ell_{\mathrm{cr}}
\end{array}\right.
$$

Note carefully that the measure of deformation $\gamma R$ having dimensions of length, this unified law is strongly sizedependent: the thicker the MWCNTs, the smaller the 
buckling shear strain. The above law also manifests itself in the data collapse for the rescaled torque-twisting angle relation shown in Fig. 1(c), which follows from Torque $=\partial E / \partial \Theta$.

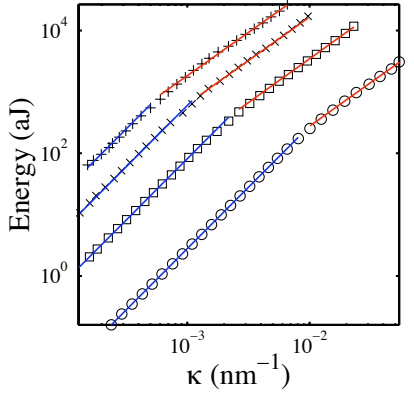

(a)

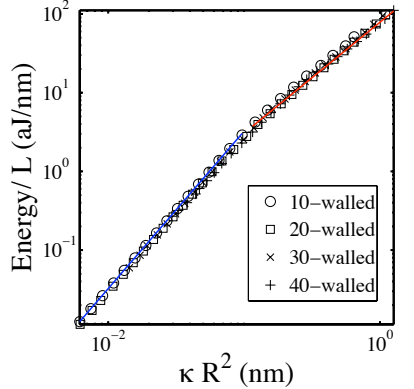

(b) (c)

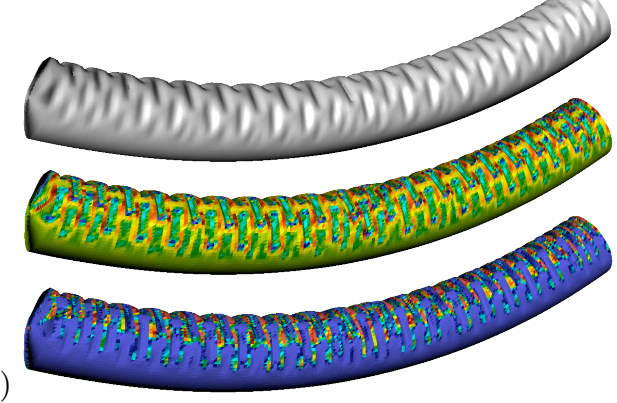

FIG. 2: Bent MWCNTs. (a) Strain energy vs curvature loglog plots for various MWCNTs and (b) data collapse upon appropriate rescaling. The power-law fits with exponents 2 (blue) and 1.42 (red) are shown for illustration. (c) 40-walled CNT in pure bending, deformed shape (top), Gaussian curvature map (middle), and energy density map (bottom). The color scales coincide with those of Fig. 1

In bending the situation is analogous. The maximum strain $\varepsilon=\kappa R$ occurs at diametrically opposed points of the outer shell with opposite signs, where $\kappa$ denotes the curvature imposed on the tube. By defining the bending spring constant $K_{b}=\pi Y_{s} / R^{2} \sum_{i=1}^{n} r_{i}^{3}$, the blue fits in Fig. 2(a) follow from $E=1 / 2 L K_{b} \varepsilon^{2}$. The classical size-invariant elastic scaling $\tilde{E}(\varepsilon)$ does not collapse the anharmonic branches of the data, while the sizedependent rescaling $(E / L)$ vs $\left(\kappa R^{2}\right)$ highlights the unified energy-curvature law, see Fig. 2(b). Again, the critical bucking strain scales as $\varepsilon_{\text {cr }} \propto R^{-1}$, and the unified law is completely described in terms of an elastic factor, here $\pi Y_{s} /(8 t)$, the anharmonic exponent, and the critical length $\ell_{\mathrm{cr}}=\kappa_{\mathrm{cr}} R^{2}$. These observations contrast with the size-independent critical buckling strain reported for graphite 2D models of MWCNTS [12, 23].

The parameters of the unified energy-deformation laws for the Brenner potential and its modified version are are reported in Table I. Strikingly, the critical length-scale is quite insensitive to the potential and the deformation mode, while the anharmonic exponent is considerably larger for twisting than for bending. While the harmonic response mobilizes either the Young's modulus (bending)
TABLE I: Parameters of the scaling law

\begin{tabular}{lcccc}
\hline \hline & \multicolumn{2}{c}{ Brenner $^{a}$} & \multicolumn{2}{c}{ Modified Brenner $^{b}$} \\
& $\ell_{\text {cr }}(\mathrm{nm})$ & $a$ & $\ell_{\text {cr }}(\mathrm{nm})$ & $a$ \\
\hline Torsion & 0.13 & 1.68 & 0.11 & 1.63 \\
Bending & 0.11 & 1.42 & 0.10 & 1.41 \\
\hline \hline
\end{tabular}

${ }^{a} Y_{s}=236 \mathrm{~J} / \mathrm{m}^{2}, G_{s}=83 \mathrm{~J} / \mathrm{m}^{2}, C_{b}=2.2 \mathrm{eV} \AA^{2} /$ atom.

${ }^{b} Y_{s}=340 \mathrm{~J} / \mathrm{m}^{2}, G_{s}=148 \mathrm{~J} / \mathrm{m}^{2}, C_{b}=3.8 \mathrm{eV} \AA^{2} /$ atom.

or the shear modulus (torsion), the anharmonic regime mixes stretch, shear, and bending of the walls. Hence the dependence of $a$ on the potential is not surprising.

Despite a full analytical understanding of complex buckling phenomena such as rippling is not available, the modern literature provides key ideas to help understand qualitatively the results above, in particular the fact that the anharmonic exponent in torsion is much larger than that in bending. In the mechanics of thin elastic sheets, isometric maps are of paramount importance because they provide deformation mechanisms that avoid in-plane stretching or shearing, sometimes at the expense of sharp folds [24]. In materials that exhibit a large inplane rigidity as compared to the bending rigidity, e.g. paper or graphene, localized regions of high curvature are often energetically favorable. Folds and conical dislocations with optimal balance between in-plane and bending energies have been characterized [10]. Recent analytical studies point out the efficiency of the Yoshimura pattern, observed in the compressed side of bent nanotubes, in achieving nearly isometric deformations for compressed sheets [11]. According to Gauss' Theorema Egregium, the Gaussian curvature of a surface is invariant with respect to isometries; since for the undeformed configuration of CNTs the Gaussian curvature vanishes, it serves as a measure of the degree of local isometry. Figure 2(c) shows a color map of the Gaussian curvature on a bent MWCNT. It can be observed that the curvature concentrates along the ridges (positive) and cones (positive and negative), while most of the surface has very small curvature. This results in a strain energy density (energy per unit area) sharply concentrated on the folds. The rippling pattern that develops in torsion is far less efficient in approximating an isometry. Figure 1(d) shows that these boundary conditions produce a deformation that is nowhere close to isometric: the helicoidal ridges have positive curvature while the furrows are negatively curved. The morphological features are not as sharp as for bending, but they cover a larger area of the surface of each wall. This results in a significantly larger portion of tube with large strain energy (note that the color scales are identical in both figures), and explains qualitatively the stiffer post-buckling response in torsion.

The scaling of the critical strain in bending, $\varepsilon_{\mathrm{cr}} \propto R^{-1}$, follows from linearized buckling analysis of cylindrical shells under specific assumptions, as noted in [2]. This reference finds excellent agreement between the critical curvature found in simulations of single-walled CNTs of 
small diameter and that predicted by the theory with $\ell_{\mathrm{cr}}=0.039 \mathrm{~nm}$. The larger critical length-scale we find is probably due to the stabilization effect provided by inner tubes to the outer shell that buckles first. Even if this agreement is comforting, it should be emphasized that these scalings of the critical strain follow from radically different approaches: here it is obtained as the transition between two regimes of the energy-deformation relation, one of which is genuinely nonlinear, while linearized buckling studies the very onset of the instability and uses hypothesis of doubtful validity for MWCNTs. In torsion, [2] reports $\gamma_{\mathrm{cr}} \propto R^{-3 / 2}$ for SWCNTs, which again follows from linearized buckling theory, in contrast with $\gamma_{\mathrm{cr}} \propto R^{-1}$ for MWCNTs found here.

We now compare the predictions of the unified law against experiments. Given the limited amount of quantitative data available in the literature, we test whether the critical length-scale characterizing the onset of rippling is consistent with observations of MWCNTs subject to deformation, for which there is evidence of rippling or its absence. In [3], a 45-walled CNT with $R=15.5 \mathrm{~nm}$ and $\kappa=1 / 400 \mathrm{~nm}^{-1}$ was observed statically to display rippling. For the same tube in oscillatory motions reaching $\kappa=1 / 1200 \mathrm{~nm}^{-1}$, indirect evidence thought the resonant frequencies indicated rippling. Our theory is consistent with these observations since in the first case $R^{2} \kappa=0.6 \mathrm{~nm}>\ell_{\text {cr }}$ and in the second $R^{2} \kappa=0.2 \mathrm{~nm}>\ell_{\mathrm{cr}}$. The same reference reports on a 12 -walled $\operatorname{CNT}(R=4 \mathrm{~nm})$ bent to $\kappa=1 / 300 \mathrm{~nm}^{-1}$ observed not to display rippling, in agreement with the theory since $R^{2} \kappa=0.053 \mathrm{~nm}<\ell_{\mathrm{cr}}$. In torsional experiments [4], shear strains between $2 \%$ and $5 \%$ for MWC-
NTs with radii in between 6 and $17.5 \mathrm{~nm}$ were reported, together with indirect signs of nonlinear mechanical behavior. The most conservative prediction with our theory, $\gamma R=0.02 \cdot 6 \mathrm{~nm}=0.12 \mathrm{~nm}$, suggests that indeed torsional rippling occurred in these experiments. In [22], a cantilevered MWCNT $(R=16.4 \mathrm{~nm})$ was loaded with an AFM at a distance of $L=813 \mathrm{~nm}$ from the fixed end, and the force-displacement was recorded. The critical rippling end deflection was measured to be of about $\delta_{\text {cr }}=150 \mathrm{~nm}$. The critical rippling curvature, occurring at the fixed end, can be computed as $\kappa_{\mathrm{cr}}=3 \delta_{\mathrm{cr}} / L^{2}$, resulting in $\ell_{\mathrm{cr}}=\kappa_{\mathrm{cr}} R^{2}=0.18 \mathrm{~nm}$, to be compared with the data reported in Table I. Given the uncertainties in this experiment [22] and in the inter-atomic potentials, we consider the agreement to be good.

We have characterized the nonlinear mechanical behavior of MWCNTs in pure bending and torsion through systematic simulations of realistically large systems. Their mechanics at the mesoscopic level are described by a size-dependent unified law consisting of two different power-law regimes. Size-dependence in materials science is usually associated with irreversible processes such as plasticity or fracture. Here, it arises as a consequence of a reversible geometric instability. The reported results are in agreement with available data, and can improve the interpretation of experiments. Our predictions can be experimentally tested, particularly if mechanical loading is supplemented by high resolution microscopy.

The support of the European Commission (MIRG-CT2005-029158 and -029178), the Ministerio de Educación y Ciencia (DPI2007-61054) and the Barcelona Supercomputing Center-CNS, is gratefully acknowledged.
[1] T. Dumitrica, M. Hua, and B. I. Yakobson, Proceedings of the National Academy of Sciences 103, 6105 (2006).

[2] B. I. Yakobson, C. J. Brabec, and J. Bernholc, Physical Review Letters 76, 2511 (1996).

[3] P. Poncharal, Z. L. Wang, D. Ugarte, and W. A. de Heer, Science 283, 1513 (1999).

[4] S. J. Papadakis, A. R. Hall, P. A. Williams, L. Vicci, M. R. Falvo, R. Superfine, and S. Washburn, Physcal Review Letters 93, 146101 (2004).

[5] M. Zhang, K. R. Atkinson, and R. H. Baughman, Science 306, 1358 (2004).

[6] A. Cao, P. L. Dickrell, W. G. Sawyer, M. N. GhasemiNejhad, and P. M. Ajayan, Science 310, 1307 (2005).

[7] S. Timoshenko and J. Gere, Theory of Elastic Stability (McGraw-Hill, New York, 1998).

[8] O. Lourie, D. M. Cox, and H. D. Wagner, Physical Review Letters 81, 1638 (1998).

[9] M. Arroyo and T. Belytschko, Physical Review Letters 91, 215505 (2003).

[10] E. Cerda, S. Chaieb, F. Melo, and L. Mahadevan, Nature 401, 46 (1999).

[11] S. Conti and F. Maggi, Arch. Rat. Mech. Anal. doi: 10.1007/s00205-007-0076-2 (2007).

[12] J. Liu, Q. Zheng, and Q. Jiang, Physical Review Letters 86, 4843 (2001).
[13] D. W. Brenner, Physical Review B 42, 9458 (1990).

[14] M. Arroyo and T. Belytschko, Physical Review B 69, 115415 (2004).

[15] L. A. Girifalco, M. Hodak, and R. S. Lee, Physical Review B 62, 13104 (2000).

[16] J. Cumings and A. Zettl, Science 289, 602 (2000).

[17] M. Arroyo and T. Belytschko, Journal of the Mechanics and Physics of Solids 50, 1941 (2002).

[18] M. Arroyo and T. Belytschko, International Journal for Numerical Methods in Engineering 59, 419 (2004).

[19] As implemented, this method cannot describe plasticity or fracture, but it can assess the loss of stability of the lattice, which never occurs in the present simulations.

[20] X. Y. Li, W. Yang, and B. Liu, Physical Review Letters 98, 205502 (2007)

[21] M. Arroyo and I. Arias, J. Mech. Phys. Solids doi:10.1016/j.jmps.2007.10.001 (2007).

[22] E. Wong, P. Sheehan, and C. Lieber, Science 277, 1971 (1997).

[23] J. Z. Liu, Q. Zheng, and Q. Jiang, Physical Review B 67, $075414(2003)$.

[24] E. L. Starostin and G. H. M. Van Der Heijden, Nature Materials 6, 563 (2007). 